\title{
LETTER
}

\section{Landscape of protein domain interactome}

\section{Dear Editor,}

Protein domain is usually defined as distinct, compact and stable protein structural unit that folds independently of other such units (Koonin et al., 2002). The majority of proteins, especially in higher organisms, contain multiple domains (Chothia, 1992). Domain view of protein evolution provides many insights into the evolution of pathways and networks, as well as into the general direction of evolution of higher organisms. Domain structure of proteins is also important in understanding protein-protein interactions, as proteins interact with each other not as complete units, but rather via their component domains. Therefore, a protein-protein interaction (PPI) network can be viewed, at higher resolution, as the domain-domain interaction (DDI) network. However the network study in the past decade was still limited to protein but barely zoomed into domain resolution. Although some DDI-based database has been established and analyzed (Stein et al., 2005; Yellaboina et al., 2011), it remains unclear that how different domains play distinct roles in the interaction network and how do they coordinate with each other functionally.

To study the domain-domain interaction (DDI) network, first we established a baseline human protein-protein interaction network composed of 19,139 individual PPIs by integrating several protein-protein interaction databases. We then mapped domains into the PPI network based on Pfam definitions (Sonnhammer et al., 1998). We used information from three databases: iPfam (Finn et al., 2005), 3did (Stein et al., 2005) and DOMINE (Yellaboina et al., 2011) to predict DDIs based on both protein sequence and protein interaction information. In total we identified 46,712 DDIs in our network (Fig. 1A), which include three types of DDls based on the property of interaction: 1) neighboring intra-DDI, the interactions between neighboring domains within one protein result from their proximity along the amino acid chain; 2) general intra-DDI, the physical interactions between domains within one protein that do not result from their proximity along the amino acid chain but from the folding in 3D space; 3) inter-DDI, physical interactions between domains in interacting proteins (Fig. 1A).

Next we analyzed the topological characteristics of DDI network. Similar to proteins in the PPI network, node degree (k) distribution of domains approximately follow power-law distribution (Fig. S1), which shows that most of the domains are linked to only few other domains. In contrast, some domains such as the "SH2" domain on Grb2 protein are connected to many other domains ( $k=122)$, which is consistent with its central role in dynamic regulation of tyrosine kinase signal, the key signal of eukaryotic cell growth (Tinti et al., 2013). Like the PPI network, the DDI network is also a scalefree network but it has significantly higher betweenness and clustering coefficient than PPI network (Fig. S2). In the DDI network, each domain is represented by multiple nodes (appears more than once) as a portion of different proteins, and each time when it appears, it may have distinct partners. This is unlike in PPI network that each protein is represented by a unique node. So in the DDI network, certain domains may show a tendency to interact with many different types of domains and can be considered as "promiscuous", or "P domain". For example, "MAM" domain has 10 interacting partners in the human DDI network, and 9 of them were different domains. This is consistent with previous report that "MAM" domain exist in many functionally diverse proteins to play different roles (Beckmann and Bork, 1993). In contrast, some domains in DDI network tend to participate in limited types (under extreme condition, only one type) of DDIs and therefore can be considered as "chaste", or " $\mathrm{C}$ domain". For instance, the "Beta-catenin-interacting protein ICAT" domain has 8 partners in DDI network and all of them are the same domain type ("Armadillo repeats"), as the ICAT domain only exist in ICAT protein whose main function is to inhibit betacatenin/TCF pathway (Graham et al., 2002). The difference between promiscuous and chaste domains is illustrated in Fig. 1B.

To define domain properties quantitatively, for each domain, we counted the types of domains it interacts with to calculate an interacting heterogeneity coefficient $\mathrm{H}$ (see the Methods section for the exact definition). The average value of $\mathrm{H}$ is equal to 0.16 and whole distribution is shown in Fig. 1C. According to the distribution, we define the domains with $\mathrm{H}>0.5$ as promiscuous domains ( $P$ domains), and those with more than one interacting partner and $\mathrm{H}<0.005$ as chaste domains ( $C$ domains). In total there are $342 \mathrm{P}$ domains and $406 \mathrm{C}$ domains defined, with the other 1448 domains sharing intermediate features of $C$ and $P$ domains. The $P$ domains and $C$ domains were found evenly distributed in intra- and inter-protein DDIs.

We further analyzed the node degrees of $P$ and $C$ domains, as shown in Fig. 1D, we found that node degree for 
A

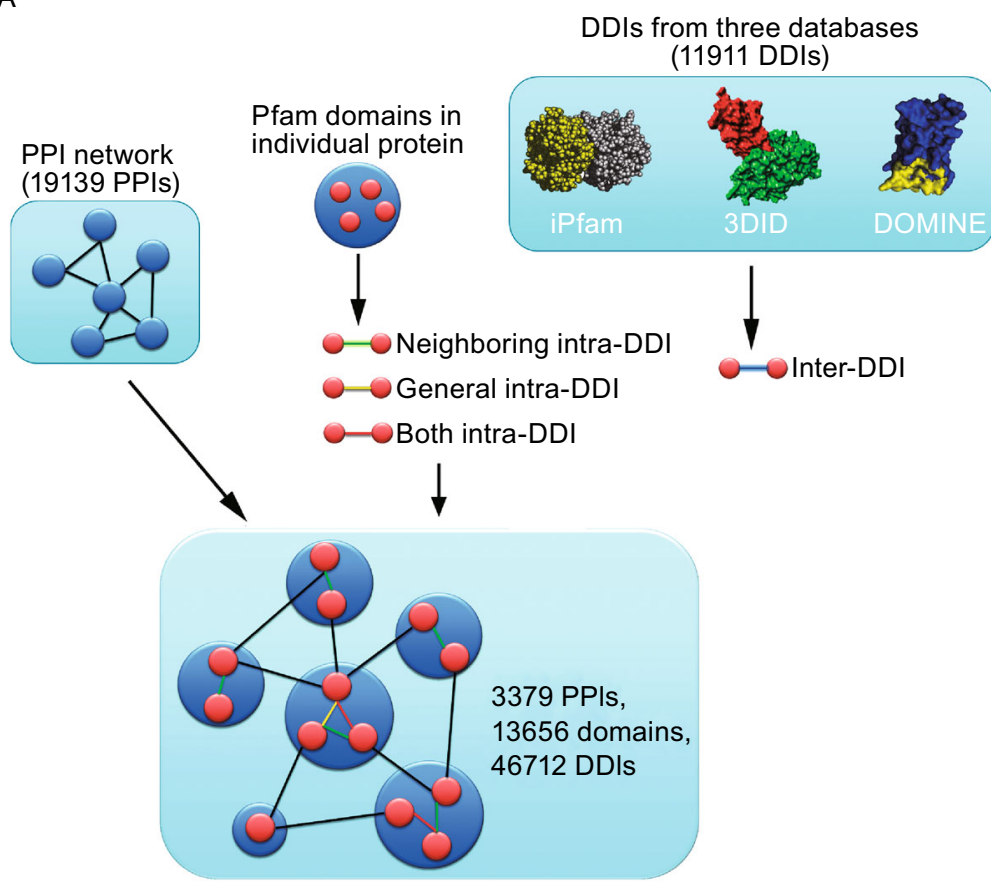

D
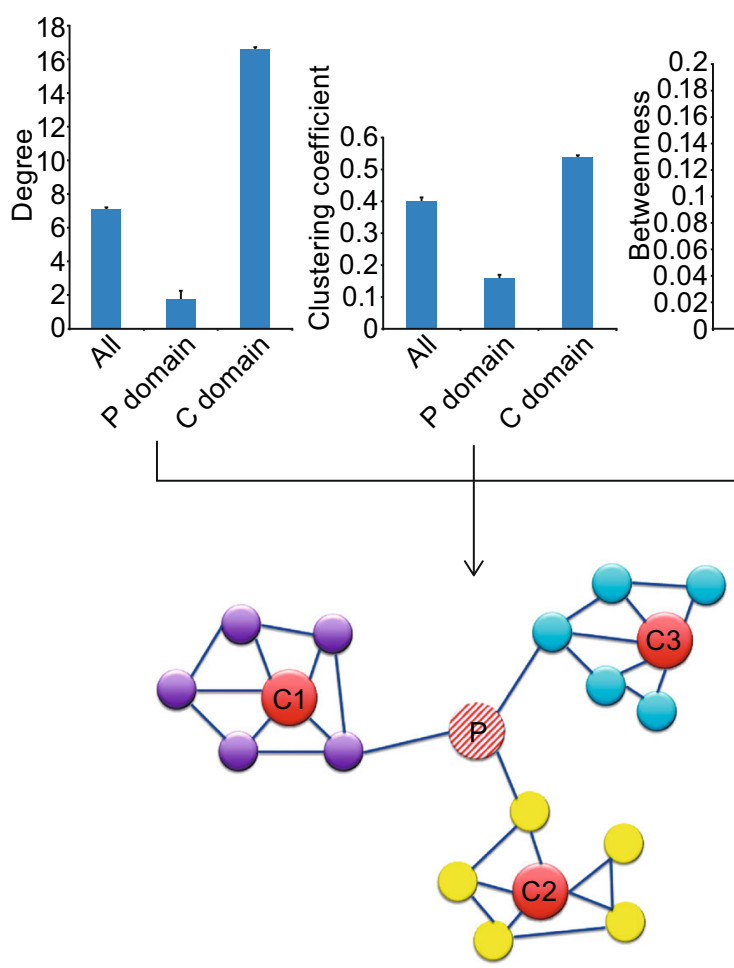

Promiscuous domain

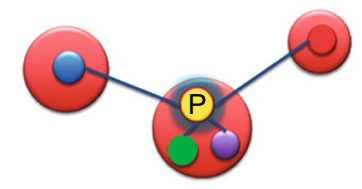

Chaste domain
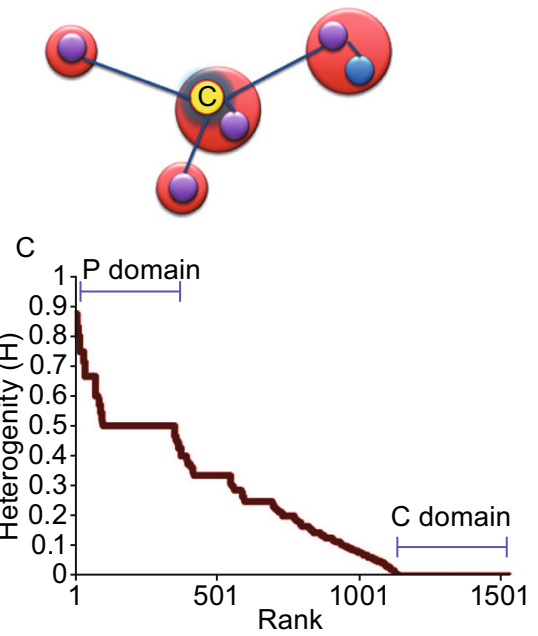

E

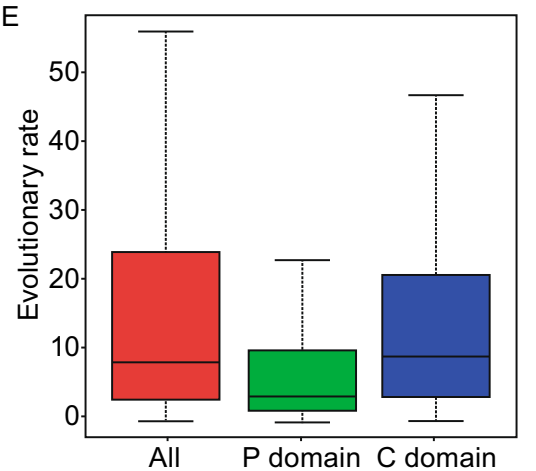


4 Figure 1. DDI network, domain promiscuity and characteristics of $P$ and $C$ domains. (A) Flowchart of constructing DDI network. (B) Definition of Promiscuous domains ( $P$ domains) and Monogamous domains ( $\mathrm{C}$ domains). The small circles indicate identical (same color) or different (various colors) domains, the large circles indicate proteins harboring single or multiple domains. (C) Identifying $\mathrm{P}$ and $\mathrm{C}$ domains in DDI network by calculating interaction heterogeneity. (D) Network characteristics of all, $\mathrm{P}$ and $\mathrm{C}$ domains. Upper panel, the degree, clustering coefficient and betweeness of all, $\mathrm{P}$ and $\mathrm{C}$ domains. Error bar indicates standard error of the mean. Lower panel, a model of $\mathrm{P}$ and $\mathrm{C}$ domain localization in network. ' $\mathrm{P}$ ' indicated $\mathrm{P}$ domain, and ' $\mathrm{C} 1 / \mathrm{C} 2 / \mathrm{C}$ ' represented $\mathrm{C}$ domain. (E) Box plots of evolutionary rates of all, $\mathrm{P}$ and $\mathrm{C}$ domains. (F) Mutation distribution on domains of different heterogeneity. The $\mathrm{P}$ domains and $\mathrm{C}$ domains corresponded to the domains with heterogeneity $\geq 0.5$ and $<0.005$ respectively.

the $\mathrm{P}$ domains are lower than average $\left(P<10^{-4}\right.$ by a Wilcoxon rank-sum test), while $C$ domains' are higher $\left(P<10^{-4}\right.$ by a Wilcoxon rank-sum test). About $30 \%$ of $C$ domains have degree $k \geq 10$. Similarly, for clustering coefficient, which measures the density of network module, $\mathrm{P}$ domains are lower than average, while $\mathrm{C}$ domains are higher. Therefore, the highly interacted $\mathrm{C}$ domains are "hubs" in the network, which function to organize the local network modules. Interestingly, the betweenness of domains, which measures the number of shortest paths between any domain pair that involves a given domain (Yu et al., 2007), is higher than average in the $\mathrm{P}$ domains and lower in the $\mathrm{C}$ domains. Therefore, $\mathrm{P}$ domains are non-hub "bottlenecks" of the network, which usually link different function modules together (Fig. 1D). These results are consistent with Gene Ontologybased function analysis using Pfam2Go, which showed that $P$ domains were enriched in $\mathrm{GO}$ terms associated with very general biological functions, such as "metabolic process", "DNA-directed RNA polymerase activity" and "nucleotidyltransferase activity" ( $P$-value $\left.<10^{-6}\right)$. In contrast, no GO terms were found to be enriched among $C$ domains, suggesting that each $\mathrm{C}$ domain may have unique, non-overlapping functions.

Some previous studies (Zmasek and Godzik, 2011) analyzed the evolution pattern of domain repertoire in eukaryotes. Here we examined whether the interacting patterns of domains could affect their evolution. We found no difference in terms of evolutionary rate between $\mathrm{C}$ domain and all other domains. However, the evolutionary rate of $P$ domains was much lower than the average (Fig. 1E, $P<10^{-4}$, Wilcoxon rank-sum test) and this effect still exists even if the difference in the contact degree was taken into effect. So the result suggests that the evolution of $P$ domains was constrained by the diversity of their interaction partners.

To identify possibly different roles of $P$ and $C$ domains in diseases, we investigated the distribution of oncogenic mutations in the DDI network. Previous reports (Wang et al., 2012) showed that disease-related mutations tend to be localized in domains linking to another protein (thereafter called "interface" domains). Here we examined the relationship between $\mathrm{H}$ and mutation rate, and found that $\mathrm{P}$ domains and $C$ domains do not have advantage to accumulate mutations. Instead, the domains with intermediate $\mathrm{H}$ values (0.02 0.5) tend to accumulate mutations (Fig. 1F, $P$ value $<10^{-4}$ by a Wilcoxon rank-sum test). Considering that $C$ domains and $\mathrm{P}$ domains are hubs and bottlenecks of the DDI network respectively, this observation suggests that oncogenic mutations tend to avoid the topologically important nodes of the biological networks, probably because such mutations in key domains would lead to immediate breakdown of the whole system so become highly deleterious for cancer cell survival.

After analyzing the $\mathrm{P}$ and $\mathrm{C}$ domains, we continued to study the pattern of DDI pairs. As each domain can appear more than once in the network, each domain pair can also appear more than once. We categorized the 46,712 DDIs into 3,445 pairs, and calculated how often they show up in the network. And we named domain pairs that appear more (or less) frequently than average as "frequent DDIs" (or "rare DDIs"). The most frequent and rare DDIs are listed in Table S1. Within the list, we noticed that the domains in frequent DDIs were functionally similar to each other; instead, domains in rare DDIs usually have different (or complementary) functions. This observation is expected as domains with similar functions tend to coordinate with each other to function together. Evolutionary rate calculation also showed that co-evolving domains (measured by Jensen-Shannon Divergence score, JSD* $\leq 0.05$ ) interact with each other more frequently (Fig. 2A), which should be due to the interacting partners are usually subjective to the identical selective pressure. However, network edge attack analysis indicated that the rare DDls were more important to maintain the network. Loss of rare DDIs rapidly increased the characteristic path length and decreased the size of largest component, indicating the rapid breakdown of the network (Fig. 2B). The result suggests that rare DDIs function by establishing unique links between different functional modules.

To understand the pattern how different biological functions are coordinated through combination of domains, we integrated the domain function information onto the DDI network. We found that there are some function combination appears more frequently than by chance. For example, in the network there are 73 domains annotated with "double-stranded RNA binding" function and 29 domains annotated with "RNA processing" function, and they form 11 function combinations with the frequency $(=0.239)$ much higher than statistically expected $(=0.066, P<0.01$ by a Wilcoxon ranksum test). This is also consistent with our knowledge that protein binding of double-stranded viral RNA and processing it are two closely related biological processes. The top 20 frequent function combinations are listed in Fig. 2C.

Furthermore, to understand the spatial distribution of domains, we mapped the subcellular location information of domains to the DDI network. We found that the 
DDI frequency between co-evolving domains

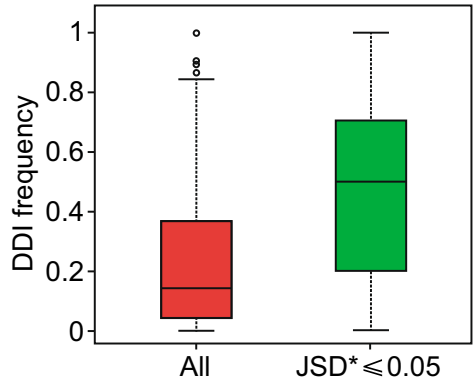

C

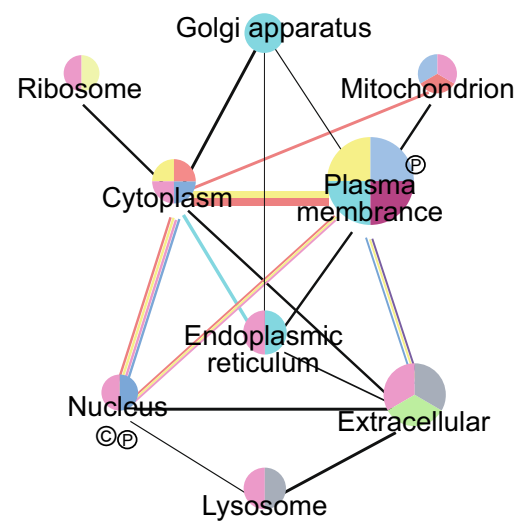

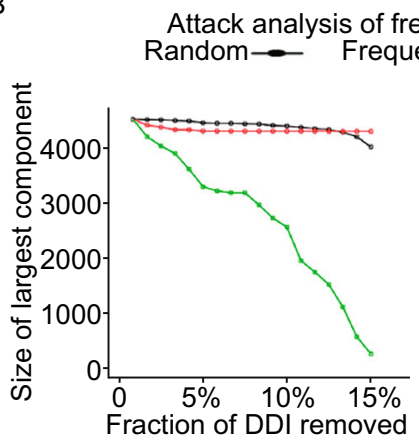

Attack analysis of frequent and rare DDIs

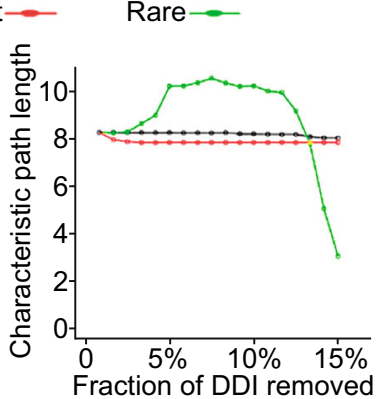

$\begin{array}{llllll}0 & 100 & 200 & 300 & 400 & 500\end{array}$ DNA binding - Transcription,DNA-dependent
Regulation of transcription,DNA-dependent - Nucleus Sequence-specific DNA binding - Steroid hormone mediated signaling pathway ATP-dependent helicase activity - Nucleic acid binding Nucleic acid binding - Helicase activity ATP binding-Mismatch repair Sequence-specific DNA binding-Sequence-specific DNA binding TF activity Nucleus - Steroid hormone mediated signaling pathway Sequence-specific DNA binding TF activity - Steroid hormone mediated signaling pathway Regulation of transcription,DNA-dependent - Zinc ion binding Protein tyrosine phosphatase activity - Protein kinase activity Sequence-specific DNA binding - Nucleus Protein dephosphorylation - Protein kinase activity Calcium ion binding - Homophilic cell adhesion Protein phosphorylation - ATP binding Homophilic cell adhesion-Membrane Zinc ion binding - Nucleus ATP binding - Helicase activity GTP binding - Signal transduction Calcium ion binding - Membrane

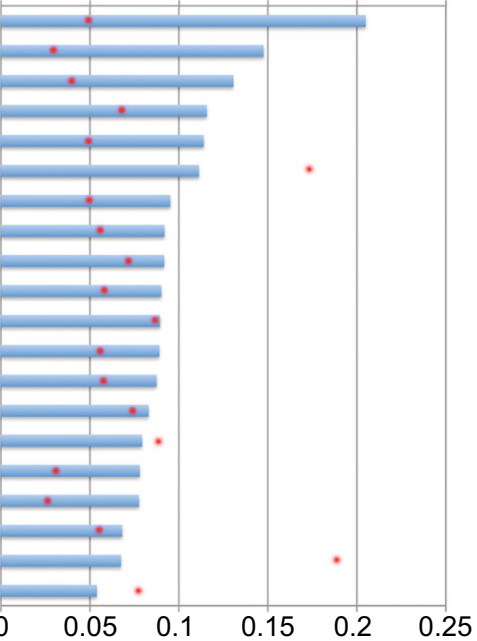

Cell communication

- Biological regulation

- Metobolic process

- Binding

- Immune system process

- Molecular transducer activity

- Localization

- Biological adhesion

- Catalytic activity

Structural molecule activity

- No enrichment

(C) Chaste domain

(P) Promiscuous domain

Figure 2. Co-evolution, attack of DDIs, function combination and subcellular localization of DDIs. (A) Box plots of frequency of appearance of all DDI pairs and co-evolving DDI pairs (JSD* < 0.05). (B) Effects of the gradual removal of randomly selected DDI, frequent DDI or rare DDI on the largest component size (upper panel) and characteristic path length (lower panel) of the network. (C) Top 20 most frequent domain function combinations (counting > 50). Bars indicated the normalized frequency (ranged from 0 to 1 ). Red dots indicated the absolute number of the function combinations in the network (ranged from 0 to 400). Redundant combinations were removed. (D) DDls distribution in subcellular components. The size of the nodes indicates the relative number of DDIs that are within a subcellular component. The thickness of the edges indicates the relative number of DDls that are between two subcellular components. The colors correspond to different $\mathrm{GO}$ functional terms. The circled $\mathrm{C}$ and $\mathrm{P}$ indicate the enrichment of $\mathrm{C}$ domain and $\mathrm{P}$ domain.

communication between domains within the following locations is most frequent: "extracellular part-plasma membrane", "plasma membrane-cytoplasm" and "cytoplasm- nucleus" (Fig. 2D). This is in consistent with our understanding that the "extracellular part-plasma membrane-cytoplasm-nucleus" is the most classical signal transduction 
axis in cells. Furthermore, we uncovered the complex relationship between domain function and domain subcellular localization. For instance, we showed that "biological adhesion" is a unique function that fulfilled by DDI within extracellular part, and "immune system process" is fulfilled by DDI between extracellular part and plasma membrane. Such observation is also consistent with biological knowledge in prior (Gilbert, 1986; Kupiec-Weglinski et al., 1993). We also found that $P$ domains are enriched in both plasma membrane and nucleus, while $\mathrm{C}$ domains are comparatively limited in nucleus. This could be explained as the domains on the cell surface (plasma membrane) need to be promiscuous to adapt to various outside environment. The analysis above altogether indicated that in higher organism, domains are functionally well combined and spatially well organized. Altogether our studies uncover the landscape of how domains interact with each other to make the whole biological system works properly.

\section{FOOTNOTES}

This work was supported by the startup grant from Tongji University and Shanghai Pulmonary Hospital. We thank Dr. Adam Godzik for helpful discussion of this work. Drs. Dana Weekes, Zhanwen Li, Laszlo Koska, Piotr Cieplak, Anna Sheydina and Padmaja Natarajan for valuable suggestions.

Ting Zhang, Shuang Li and Wei Zuo declare that they have no conflict of interest. This article does not contain any studies with human or animal subjects performed by the any of the authors.

Ting Zhang ${ }^{2 \bowtie}$, Shuang $\mathrm{Li}^{3}$, Wei Zuo ${ }^{1 凶}$

${ }^{1}$ Shanghai Pulmonary Hospital, School of Medicine, Tongji University, Shanghai 200092, China

${ }^{2}$ Genome Institute of Singapore, A*STAR, Singapore 138672, Singapore

3 Tianjin International Joint Academy of Biomedicine, Tianjin 300457, China

$\bowtie$ Correspondence: zhangt1@gis.a-star.edu.sg (T. Zhang), zuow@tongji.edu.cn (W. Zuo)

\section{OPEN ACCESS}

This article is distributed under the terms of the Creative Commons Attribution 4.0 International License (http://creativecommons.org/ licenses/by/4.0/), which permits unrestricted use, distribution, and reproduction in any medium, provided you give appropriate credit to the original author(s) and the source, provide a link to the Creative Commons license, and indicate if changes were made.

\section{REFERENCES}

Beckmann G, Bork P (1993) An adhesive domain detected in functionally diverse receptors. Trends Biochem Sci 18:40-41

Chothia C (1992) Proteins. one thousand families for the molecular biologist. Nature 357:543-544

Finn RD, Marshall M, Bateman A (2005) iPfam: visualization of protein-protein interactions in PDB at domain and amino acid resolutions. Bioinformatics 21:410-412

Gilbert SF (1986) Cell surface receptors in development and immunity: a speculative review. Dev Comp Immunol 10:279-284

Graham TA, Clements WK, Kimelman D, Xu W (2002) The crystal structure of the beta-catenin/ICAT complex reveals the inhibitory mechanism of ICAT. Mol Cell 10:563-571

Koonin EV, Wolf YI, Karev GP (2002) The structure of the protein universe and genome evolution. Nature 420:218-223

Kupiec-Weglinski JW, Heemann UW, Coito AJ, Tullius SG, Tilney NL, de Sousa M (1993) Adhesion molecule interaction with extracellular matrix. Exp Nephrol 1:78-82

Sonnhammer EL, Eddy SR, Birney E, Bateman A, Durbin R (1998) Pfam: multiple sequence alignments and HMM-profiles of protein domains. Nucleic Acids Res 26:320-322

Stein A, Russell RB, Aloy P (2005) 3did: interacting protein domains of known three-dimensional structure. Nucleic Acids Res 33: D413-D417

Tinti M, Kiemer L, Costa S, Miller ML, Sacco F, Olsen JV, Carducci M, Paoluzi S, Langone F, Workman CT et al (2013) The SH2 domain interaction landscape. Cell Rep 3:1293-1305

Wang X, Wei X, Thijssen B, Das J, Lipkin SM, Yu H (2012) Threedimensional reconstruction of protein networks provides insight into human genetic disease. Nat Biotechnol 30:159-164

Yellaboina S, Tasneem A, Zaykin DV, Raghavachari B, Jothi R (2011) DOMINE: a comprehensive collection of known and predicted domain-domain interactions. Nucleic Acids Res 39: D730-D735

Yu H, Kim PM, Sprecher E, Trifonov V, Gerstein M (2007) The importance of bottlenecks in protein networks: correlation with gene essentiality and expression dynamics. PLoS Comput Biol 3 : e59

Zmasek CM, Godzik A (2011) Strong functional patterns in the evolution of eukaryotic genomes revealed by the reconstruction of ancestral protein domain repertoires. Genome Biol 12:R4
Electronic supplementary material The online version of this article (doi:10.1007/s13238-015-0158-0) contains supplementary material, which is available to authorized users. 